\title{
Resonance shape analysis of neutron capture measurements from xenon spherical samples
}

\author{
O. Litaize ${ }^{1, a}$, G. Noguere ${ }^{1}$, C. de Saint Jean ${ }^{1}$, P. Mutti ${ }^{2}$, A. Brusegan ${ }^{3}$, and F. Corvi ${ }^{3}$ \\ 1 CEA-Cadarache, 13108 Saint-Paul-lez-Durance, France \\ 2 Institute Laue Langevin, BP. 156, 38042 Grenoble Cedex 9, France \\ 3 Institute for Reference Materials and Measurements, Joint Research Center, Retieseweg, 2440 Geel, Belgium
}

\begin{abstract}
Techniques have been developed in the frame of the CONRAD Nuclear data analysis code in order to take into account the multiple scattering correction for capture yields. The resonance shape analysis of these capture data have to account for these corrections during the fitting procedure. Analytical formulae and Monte Carlo simulations can be performed through Multiple-Scattering-Correction module in the CONRAD code in order to estimate the differential and total capture yields. Monte Carlo method accounts for usual biasing techniques (implicite capture and Russian roulette). This experimental correction procedure is checked against other codes like REFIT and SAMMY which are extensively used for cylindrical sample analysis. In this work, xenon spherical samples have been studied. Capture and transmission measurements of gaseous samples have been performed at the GELINA facility of the Institute for Reference Materials and Measurements (IRMM) by P. Mutti, F. Corvi and A. Brusegan. For the capture measurements, spherical samples with diameter of $8 \mathrm{~cm}$ were used. Due to the spherical shape of the sample, equivalent cylinder $(\mathrm{h} \approx 5.33 \mathrm{~cm}$ ) was taken into account in SAMMY or REFIT analysis (spherical model is not implemented in these codes). These results are compared with analysis involving multiple scattering correction for spherical targets available in CONRAD.
\end{abstract}

\section{Introduction}

The so-called multiple scattering effect is one of the major experimental correction that affect the shape of the resonances observed in the capture data measured with the time-of-flight technique. This correction is accounted for cylindrical samples in the analysis code SAMMY [1] and discussed in ref. [2]. The results provided by the SAMMY and REFIT [3] codes are compared in ref. [4].

The analytical treatment for spherical samples is not available in these two codes. The capture measurements on natural xenon performed at the IRMM by P. Mutti et al. [5] give us the opportunity to investigate the multiple scattering correction for thick spherical samples. The xenon data were used to normalize a serie of krypton data measured under the same experimental conditions. Parallel transmission measurements were performed and analysed up to $300 \mathrm{eV}$ by A. Brusegan [6]. These results were used to investigate the effect of the resolution function in transmission measurements [4].

The present work aims to analyse simultaneously the transmission and capture data by using an appropriate multiple scattering correction. This paper also described an "optimized" approach that consists to mix mathematical descriptions and improved Monte Carlo simulations for cylindrical or spherical samples. These experimental corrections were written in $\mathrm{c}++$ under NetBeans development tool and integrated in the package Experiment of the CONRAD code (see ref. [7] for a detailed presentation of the code).

Preliminary results show good agreement between codes for thin samples but some discrepancies still remain for thick spherical samples between analysis and experimental data.

\footnotetext{
${ }^{a}$ Presenting author, e-mail: olivier.litaize@cea.fr
}

\section{Capture data analysis}

The capture yield $Y(E)$ can be decomposed in a simple sum of the primary capture yield $Y_{0}(E)$ (the first interaction of the neutron in the sample is a capture), the single scattering correction $Y_{1}(E)$ (neutron is captured after one scattering) and the double-plus scattering correction $Y_{2}(E)$ (neutron is captured after two or more scatterings in the sample). This total capture yield can be factorized with $Y_{0}(E)$ as following:

$$
Y(E)=Y_{0}(E)+Y_{1}(E)+Y_{2}(E)=(1+\alpha(E)) Y_{0}(E) .
$$

Capture yields can be obtained through mathematical formula and/or estimated by a Monte Carlo method. The most important contribution to the total capture yield $Y(E)$ is the primary capture yield $Y_{0}(E)$. This quantity is related to the capture probability after a single track length $z$ in the sample:

$$
P_{c}(E, z) d z=e^{-\Sigma_{t}(E) z} \Sigma_{c}(E) d z
$$

where $\Sigma_{t}$ and $\Sigma_{c}$ are respectively the total and capture macroscopic cross sections of the medium. The primary capture yield which is also defined as self-shielding correction is then defined by:

$$
Y_{0}(E)=\int_{0}^{X} P_{c}(E, z) d z
$$

where $X$ is the maximum track length available in the target before capture without any scattering.

The single scattering correction $Y_{1}(E)$ which refers to the neutron capture after a single scattering is related to the product of the scattering probability along direction $\Omega$ within $d \Omega$ after a track $z$ at energy $E$ and the capture probability after un track $q$ at scattered energy $E^{\prime}: P_{s}(E, z) P(\Omega) P_{c}\left(E^{\prime}, q\right) d q d z d \Omega$. 
The theoretical expression for the single scattering correction is then given by equation (4):

$$
Y_{1}(E)=\int_{-1}^{1} d \mu \int_{0}^{X} d z e^{-\Sigma_{t}(E) z} \Sigma_{s}(E, \mu) \int_{0}^{Q} d q e^{-\Sigma_{t}\left(E^{\prime}\right) q} \Sigma_{c}\left(E^{\prime}\right) .
$$

The different quantities appearing in the previous formula are described here after. The neutron energy after scattering (elastic or discret inelastic) $E^{\prime}$ is given by:

$$
E^{\prime}=\frac{E}{(A+1)^{2}}\left(1+2 \gamma(E) \mu_{c m}+\gamma(E)^{2}\right)
$$

in which $A$ stands for the mass of the target nucleus divided by the mass of the neutron, $\mu_{c m}$ the cosine of the scattering angle in the center of mass system.

$$
\gamma(E)=A \sqrt{1-\frac{A+1}{A}|Q|} \stackrel{\text { el.scatt. }}{=} A
$$

where $|Q|$ is the threshold of a discret inelastic scattering (not considered if this case).

$$
\Sigma_{s}(E, \mu)=\Sigma_{s}(E) p(\mu)=\Sigma_{s}(E) p\left(\mu_{c m}\right)\left|\frac{d \mu_{c m}}{d \mu}\right| .
$$

The angular distribution is uniform in the center of mass system $\left(p\left(\mu_{c m}\right)=0.5\right)$ and then an anisotropy is taken into account in the laboratory system. The cosine of the scattering angle in the laboratory system $\mu$ is simply related to the cosine in the center of mass system $\mu_{c}$ by:

$$
\mu=\frac{1+\gamma(E) \mu_{c m}}{\sqrt{1+\gamma(E) \mu_{c m}+\gamma(E)^{2}}} .
$$

Finally the $Q$ upper boundary value for variable $q$ in equation (4) is the maximum track length available in the target after primary scattering and depend on the geometry. The double-plus scattering correction $Y_{2}$ (capture yield after two or more scatterings in the medium) is generally described under simplifying assumptions. The mathematical description of this quantity is not yet implemented in CONRAD but is estimated by a Monte Carlo module. In this module, neutrons are tracked and forced to scatter (under weight adjustments to account for capture probability) until they go outside the sample or they are killed by Russian roulette (biasing) if their own statistical weight is too low. Note that in case of a beam profile (extended source), these expressions of capture yields mentionned above, have to be multiplied by $\iint d x d y / S$ where $S$ is the source area.

\subsection{Cylindrical target}

If the target is a cylinder and if the beam axis is parallel to the cylinder axis, the total length $X$ is equal to the length $L$ of the cylinder and equation (3) gives:

$$
Y_{0}(E)=\left(1-e^{-\Sigma_{t}(E) L}\right) \frac{\Sigma_{\gamma}(E)}{\Sigma_{t}(E)}
$$

where $\Sigma_{t}$ and $\Sigma_{\gamma}$ are respectively the macroscopic total and capture cross sections of the medium. For single scattering

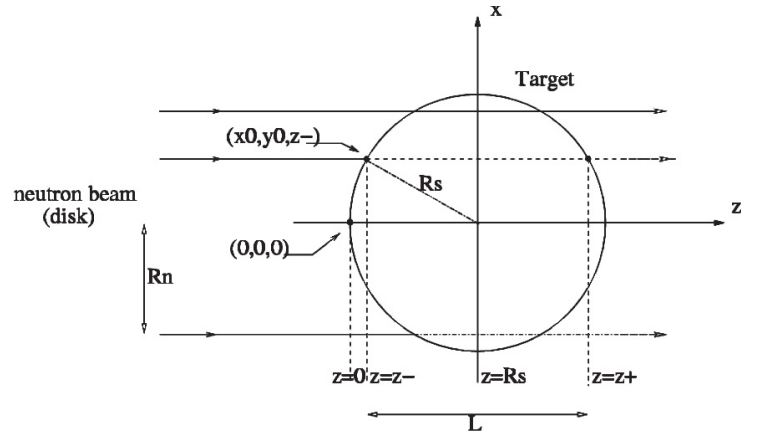

Fig. 1. Variable definition for capture yield in a spherical target.

correction $Y_{1}(E)$, the upper boundary of integration over $z$ remain $L$ i.e. the cylindrical length. The $Q$ upper boundary of the integration over $q$ only depends on the track length $z$ before scattering and the scattering angle cosine $\mu$ for infinite slabs and cylindrical samples with $R_{s} \gg R_{n}$ (sample radius few times higher than neutron beam radius): $Q(z, \mu)=-z / \mu$ for $\mu<0$. and $Q(z, \mu)=(L-z) / \mu$ for $\mu \geq 0$. Under these asumptions, the development of equation (4) give the same equations than those written in the SAMMY code user-guide [1]. In other cases (where edge effects are important) the mathematical description of the single scattering correction depends on the geometry of the target (radius $R_{s}$ and length $L$ ) and the neutron characteristics: $\mu, \phi$ respectively the polar and azimuthal angles of scattered neutrons, $z$ the track length before scattering and the radial position of the neutron in $x, y$ plan at the collision site. For instance, this $Y_{1}(E)$ correction can be represented by a fourth dimensional integral stored within a tabulated array like in the SAMMY code for instance. This development is still in progress in CONRAD and consequently, if edges effects are important, the correction is estimated by a Monte Carlo simulation. The double-plus scattering correction is also simulated.

\subsection{Spherical target}

If the target is a sphere, the maximum track length inside the sample depends on the position of the neutron entering the sphere : $X=z_{+}-z_{-}$where $\left.z_{ \pm}=R_{s} \pm \sqrt{(} R_{s}^{2}-x_{0}^{2}-y_{0}^{2}\right) .\left(x_{0}\right.$, $\left.y_{0}\right)$ is the initial position of the neutron and $R_{s}$ is the sample radius (see fig. 1).

For a spherical target, equation (3) gives:

$$
Y_{0}(E)=\int_{0}^{R_{n}} d_{z-}\left(1-e^{-2 \Sigma_{t}(E)\left(R_{s}-z_{-}\right)}\right) \frac{\Sigma_{\gamma}(E)}{\Sigma_{t}(E)} .
$$

Figure 2 shows the difference between theoretical primary capture yield for a sphere (natural xenon) and for an equivalent cylinder (same normalization). At that time, the self-shielding correction is the only theoretical correction implemented for sphere targets: the total capture yield is the sum of theoretical $Y_{0}(E)$ and simulated $Y_{1}(E)+Y_{2}(E)$.

\section{Thin gold sample}

A capture experiment was performed with a thin gold foil $(0.01 \mathrm{~cm}$-thick, radius of $4 \mathrm{~cm})$ located at $30 \mathrm{~m}$ from the 


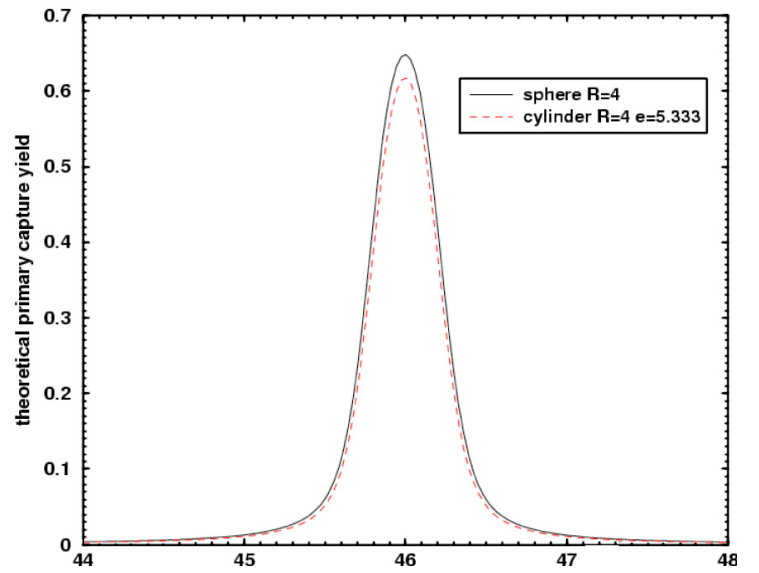

Fig. 2. Theoretical primary capture yield of the $46 \mathrm{eV}$ resonance of ${ }^{131} \mathrm{Xe}$ in sphere (higher curve) and cylinder (lower curve) targets calculated with CONRAD.

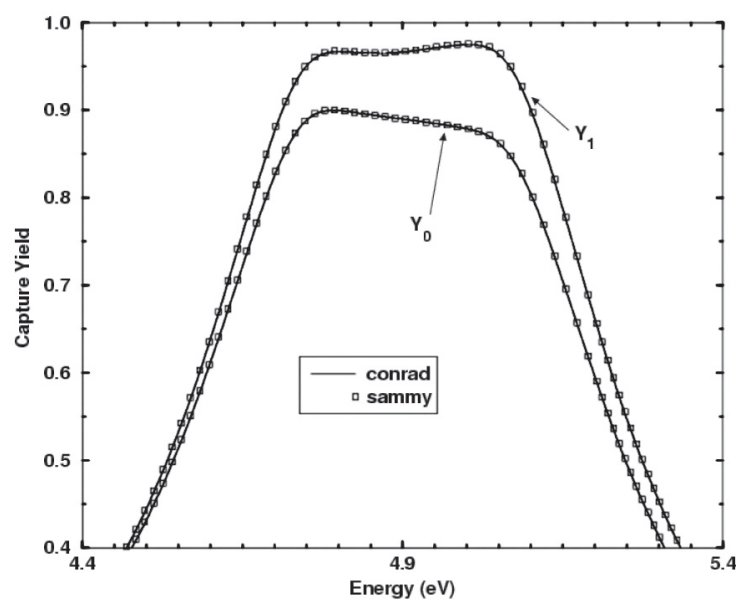

Fig. 3. Primary and secondary capture yields in a thin gold sample. Comparison between SAMMY (boxes) and CONRAD (solid curves).

neutron source. Capture gamma-rays were detected by two $C_{6} D_{6}$ liquid organic scintillators. These data were used to validate the single scattering correction implemented in CONRAD code against SAMMY and REFIT codes which were already extensively checked in previous works $[2,4]$. In figure 3 the primary capture yield and the single scattering correction are compared between SAMMY and CONRAD. For this reference thin cylindrical case, the agreement is excellent.

Figure 4 shows the consistency between the total capture yield calculated with CONRAD (dashed line), SAMMY (solid curve) and REFIT (circles) compared to the measurement (black points).

The $4.9 \mathrm{eV}$ resonance of ${ }^{197} \mathrm{Au}$ is a standard to normalize capture yield spectra with the so-called saturated resonance method [8]. Table 1 summarizes the value of this normalization obtained with REFIT, SAMMY (with multiple scattering corrections), and SAMMY (with multiple scattering corrections derived from the CONRAD code). The results are consistent and allow us to start the analysis of the natural xenon data.

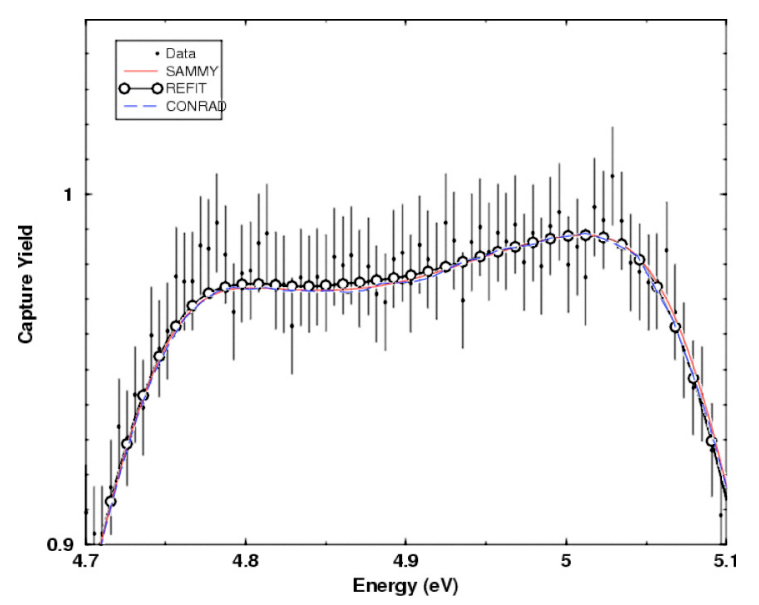

Fig. 4. Capture yield comparison between data (black points), SAMMY (solid curve), REFIT (circles) and CONRAD (dashed line) for a thin gold sample.

Table 1. Normalisation of the capture yield obtained with the first $4.9 \mathrm{eV}$ resonance of ${ }^{197} \mathrm{Au}$.

\begin{tabular}{llll}
\hline Energy Range & REFIT & $\begin{array}{l}\text { SAMMY } \\
\text { Full Mult. } \\
\text { Scatt. }\end{array}$ & $\begin{array}{l}\text { SAMMY } \\
\text { with CONRAD } \\
\text { Corrections }\end{array}$ \\
\hline$[4.8-5.0] \mathrm{eV}$ & $0.5471(5)$ & $0.5469(5)$ & $0.5456(5)$ \\
\hline
\end{tabular}

\section{Xenon data analysis in thick spherical sample}

A series of capture and transmission measurements in thick spherical samples of xenon were performed at the IRMM. A description of the experimental set-up is given in ref. [5].

The capture measurements on natural xenon spherical samples is a typical case of thick target. The difficulties compared to usual thin cylindrical targets are the geometry (sphere compared to cylinder), the thichness (thick compared to thin) and the material (multiple isotopes compared to single isotope). This is definitively not an ideal case. Fortunately, these samples are made of gazeous xenon isotopes and scattering probability in association with atomic density lead to a double-plus scatterings contribution of about less than $1 \%$. One can observe in figure 5 that the equivalent cylinder $(\mathrm{h} \approx$ $5.33 \mathrm{~cm}$ ) used in REFIT with an adapted normalization for the analysis of these capture measurements gives almost the same results than those obtained with CONRAD using the actual spherical target geometry. Nevertheless a discrepancy which is not due to the cylindrical model assumption still remain between the analysis and the data. Figure 6 shows that the capture yield is almost equal to the primary capture yield for xenon in spherical sample and the single scattering correction only contributes in the resonance wings. Because the results obtained with the REFIT, SAMMY and CONRAD codes are quite consistent and in order to perform the complete analysis simultaneously for capture and transmission data, the REFIT code was used. Propagation of errors was performed with the MCFIT tool [9] which accounts for uncertainties on sample thickness, flight length, initial delay, resolution, temperature, beam and sample diameters. The resonance parameters 


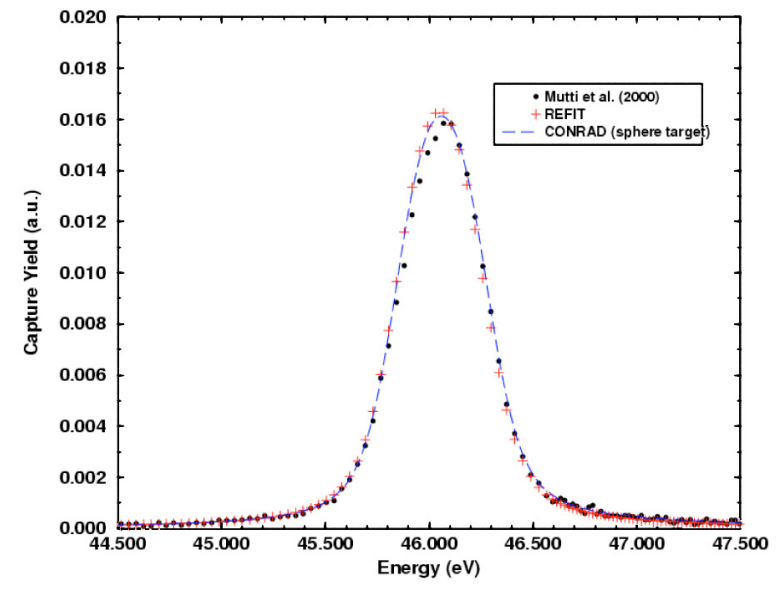

Fig. 5. Analysis of the $46 \mathrm{eV} \mathrm{J}{ }^{\pi}=2^{+}$resonance in ${ }^{131} \mathrm{Xe}$. Comparison between data (circles), REFIT with cylindrical target configuration (crosses) and CONRAD with actual spherical target (dashed line).

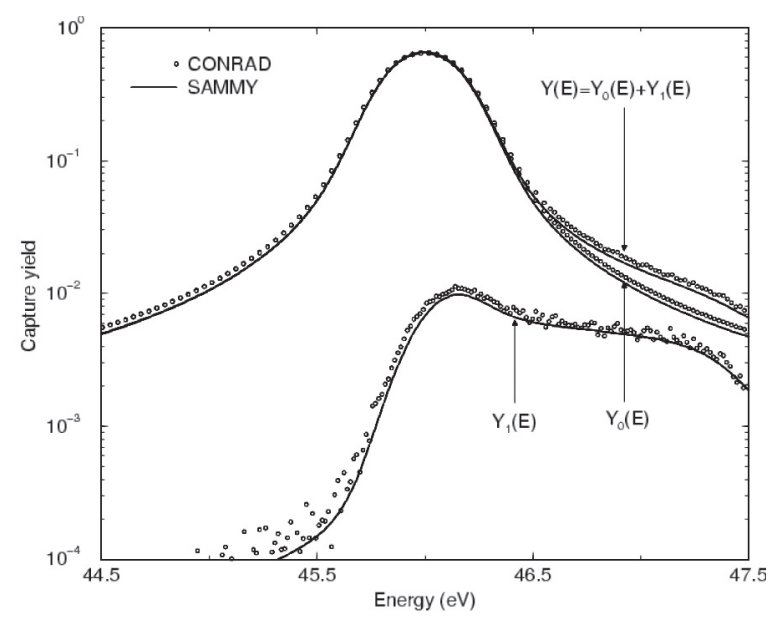

Fig. 6. Capture yield calculated with CONRAD (actual spherical sample) and SAMMY (equivalent cylindrical sample). $Y_{1}$ only contributes to the resonance wings.

derived from the fitting procedure are presented in table 2 for the three resonances of ${ }^{131} \mathrm{Xe}$ at $46 \mathrm{eV}, 75.6 \mathrm{eV}$ and $115 \mathrm{eV}$ with $l=0, J^{\pi}=2^{+}$. We obtain a consistent normalisation value for the capture yield: $N=38.61 \pm 0.43$.

The resonance parameters obtained in this work are in agreement with the recommended values published by Mughabghab [10] and those ones used in JEFF-3.1. For the $115 \mathrm{eV}$ resonance, the discrepancy is larger than for other resonances but again the result lies in the confident interval.

\section{Future work}

The work on mathematical descriptions of differential capture yields has to be continued.
Table 2. ${ }^{131} \mathrm{Xe}\left(J^{\pi}=2^{+}\right)$resonance parameters. $\mathrm{E}$ is the resonance energy, $\Gamma_{\gamma}$ stands for the radiation width and $\Gamma_{n}$ the neutron width.

\begin{tabular}{llll}
\hline \hline & Mughabghab & JEFF-3.1 & This Work \\
\hline $\mathrm{E}(\mathrm{eV})$ & $46.0 \pm 0.5$ & 46 & $46.06 \pm 0.03$ \\
$\Gamma_{\gamma}(\mathrm{meV})$ & $114 \pm 10$ & 114 & $110.6 \pm 2.5$ \\
$\Gamma_{n}(\mathrm{meV})$ & $10.9 \pm 0.8$ & 11.2 & $11.2 \pm 0.2$ \\
\hline$E(\mathrm{eV})$ & $75.58 \pm 0.08$ & 76 & $75.66 \pm 0.06$ \\
$\Gamma_{\gamma}(\mathrm{meV})$ & $123 \pm 16$ & 120 & $129.0 \pm 3.2$ \\
$\Gamma_{n}(\mathrm{meV})$ & $11.2 \pm 0.8$ & 11.2 & $11.3 \pm 0.1$ \\
\hline$E(\mathrm{eV})$ & $114.93 \pm 0.12$ & 115 & $115.0 \pm 0.1$ \\
$\Gamma_{\gamma}(\mathrm{meV})$ & $114 \pm 15$ & 114 & $125.7 \pm 3.4$ \\
$\Gamma_{n}(\mathrm{meV})$ & $31.2 \pm 1.6$ & 31.2 & $31.7 \pm 0.4$ \\
\hline \hline
\end{tabular}

A specific 'in flight' estimator could be implemented in the Monte Carlo module involved in the Multiple scattering correction procedure as developped for neutron albedos [11]. This estimator is a kind of deterministic biasing performed "in flight" during the simulation of a neutron history and allows to improve the Figure of Merite of the simulation.

Differences between cylindrical and spherical models have to be carrefully checked.

The discrepancy which is observed between analysis and data (whatever the model may be) for capture yield in spherical samples has to be checked against the following aspects: multiple scattering corrections, $\mathrm{AlMg}_{3}$ sample container, prompt background or weighting function associated with $C_{6} D_{6}$ measurements. Statistical uncertainties have to be taken into account in the Monte Carlo module in order to propagate correctly the errors during the fitting procedure.

\section{References}

1. N.M. Larson, Oak Ridge National Laboratory Report ORNL/TM-9179/R5, 2002.

2. N.M. Larson, K.N. Volev, in Proceedings of the International Conference on the New Frontiers of Nuclear Technology Reactor Physics, PHYSOR2002, Seoul, Korea, 2002.

3. M.C. Moxon, Harwell Laboratory Technical Report AEA-IN Tec-0470, 1991.

4. G. Noguere, CEA/DEN/CAD Report No. CEA-R-6071, 2005.

5. P. Mutti, H. Beer, A. Brusegan, F. Corvi, R. Gallino, in Proceedings of the International Conference on Nuclear Data for Science and Technology ND2004, Santa Fe, 2004, edited by R.C. Haight et al. (American Institute of Physics 0-7354-0254-X/05), p. 1327.

6. A. Brusegan (2000) (private communication).

7. C. De Saint Jean, B. Habert, O. Litaize, G. Noguere, C. Suteau (these proceedings).

8. R.L. Macklin, J. Halperin, R.R. Winters, Nucl. Instrum. Meth. 96, 509 (1971).

9. G. Noguere et al. (these proceedings).

10. S.F. Mughabghab, Atlas of Neutron Resonances (Elsevier, 2006).

11. O. Litaize, C.M. Diop, J.C. Nimal, J. Nucl. Sci. Technol., Suppl. 1, 507 (2000). 